\title{
National Epidemiology and Evolutionary History of Four Hand, Foot and Mouth Disease-Related Enteroviruses in China from 2008 to 2016
}

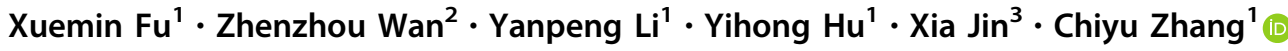

Received: 18 June 2019/Accepted: 27 August 2019/Published online: 29 October 2019

(c) Wuhan Institute of Virology, CAS 2019

\begin{abstract}
Hand, foot and mouth disease (HFMD) is a major public health concern in China. The most predominant enteroviruses that cause HFMD have traditionally been attributed to enterovirus A71 (EVA71) and coxsackievirus A16 (CVA16). Since its first large outbreak in 2008, the dominant HFMD pathogens are constantly changing. In 2013 and 2015, CVA6 exceeded both EVA71 and CVA16 to become the leading cause of HFMD in some provinces. However, there still lacks a comprehensive overview on the molecular epidemiology and evolution of HFMD-related enteroviruses at the national level. In this study, we performed systematic epidemiological analyses of HFMD-related enteroviruses using the data of 64 published papers that met the inclusion criteria, and conducted phylogenetic analyses based on 12,080 partial VP1 sequences identified in China before 31st June 2018. We found that EVA71 prevalence has decreased sharply but other enteroviruses have increased rapidly from 2008 to 2016 and that one subtype of each enterovirus is represented during the epidemic. In addition, four genotypes EVA71_C4, CVA16_B1, CVA6_D and CVA10_C are the most predominant enterovirus strains and collectively they cause over $90 \%$ of all HFMD cases in China according to the phylogenetic trees using representative partial VPI sequences. These four major enterovirus genotypes have different geographical distributions, and they may cocirculate with other genotypes and serotypes. These results suggest that more molecular epidemiological studies should be performed on several enteroviruses simultaneously, and such information should have implications for virological surveillance, disease management, vaccine development and policy-making on the prevention and control of HFMD.
\end{abstract}

Keywords Enterovirus $\cdot$ Hand, foot and mouth disease (HFMD) - Molecular epidemiology $\cdot$ Evolution · Genotype

Electronic supplementary material The online version of this article (https://doi.org/10.1007/s12250-019-00169-2) contains supplementary material, which is available to authorized users.

Chiyu Zhang

zhangcy1999@ips.ac.cn

1 Pathogen Discovery and Big Data Center, CAS Key Laboratory of Molecular Virology and Immunology, Institut Pasteur of Shanghai, Chinese Academy of Sciences, University of Chinese Academy of Sciences, Shanghai 200031, China

2 Medical Laboratory of Taizhou Fourth People's Hospital, Taizhou 225300, China

3 Viral Disease and Vaccine Translational Research Unit, CAS Key Laboratory of Molecular Virology and Immunology, Institut Pasteur of Shanghai, Chinese Academy of Sciences, Shanghai 200031, China

\section{Introduction}

Hand, foot and mouth disease (HFMD) is a self-limiting illness commonly seen in children, caused by infection with non-polio enteroviruses of the Picornaviridae family. It is clinically characterized by fever, skin eruptions on hands and feet, and vesicles in mouth (Ooi et al. 2010). Since its first outbreak in 2008 in Fuyang city, Anhui Province, HFMD has become a major public health concern in China. It was classified as a category III notifiable infectious disease on 2nd May 2008, and being monitored by a national epidemiology surveillance system in the same year. By 2018, a total of 20,537,199 HFMD cases, including 3667 deaths, had been reported by China Center for Disease Control and Prevention (CDC).

Most early HFMD cases were caused by enterovirus A71 (EVA71) and coxsackievirus A16 (CVA16), and the vast majority of severe (60\%) and fatal cases (90\%) were associated with EVA71 infection (Liu et al. 2015). 
Therefore, at the beginning, the national virological surveillance system for HFMD-related enteroviruses mainly focused on EVA71 and CVA16 using specificqPCR assays. Several years later, additional detection of other enteroviruses was performed using a PCR assay with universal primers in some regions (Xing et al. 2014; Liu et al. 2015; Wang et al. 2017; Yang et al. 2017; Mao et al. 2018). These new data showed an increasing prevalence of enteroviruses other than EVA71 and CVA16 (Lu et al. 2012; He et al. 2013; Li J et al. 2014; Li Y et al. 2018; Xu et al. 2015; Zhang et al. 2015; Fu et al. 2019). At the national level, however, there lacks a comprehensive overview on the molecular epidemiology and evolution of HFMD-related enteroviruses.

Viral genetic diversity influences virus transmission and epidemics (Vignuzzi et al. 2006; Anh et al. 2018; Wang C et al. 2018). In the past decade, a large number of molecular epidemiological investigations of HFMD were conducted on EVA71 and CVA16 (Li et al. 2005; Mao et al. 2010; De et al. 2011; Liu et al. 2014). Recent molecular epidemiological data reported an increasing prevalence of CVA6 and CVA10 (Xu et al. 2015; Zhang et al. 2015; Song et al. 2017; Ji et al. 2018; Li J et al. 2018). In particular, CVA6 cases appeared to have exceeded EVA71 cases in some regions in 2013 and 2015 ( Lu et al. 2012; He et al. 2013; Li J et al. 2014; Li Y et al. 2018; Bian et al. 2015; Xu et al. 2015; Song et al. 2017). These imply that CVA6 and CVA10 have joined EVA71 and CVA16 to be the predominant enteroviruses responsible for HFMD in China (He et al. 2013; Xu et al. 2015; Li $\mathrm{J}$ et al. 2018; Li Y et al. 2018). However, the genetic diversity characteristics and the evolutionary history of these four main HFMD-related enteroviruses (EVA71, CVA16, CVA6 and CVA10) are poorly understood. Furthermore, China has large geographic areas with different seasonal patterns of HFMD incidence from North to South (Xing et al. 2014; Mao et al. 2018). Whether certain enteroviruses, or genotypes of the same enterovirus are linked to different HFMD epidemic patterns remains unclear.

To address these issues, we performed meta-analyses on cohorts which have molecular epidemiological and enterovirus sequence data available in the public databases. Our results present a comprehensive overview of the recent national epidemiology and evolutionary history of the major enteroviruses that cause HFMD in China.

\section{Materials and Methods}

\section{Collection of Molecular Epidemiological Data on HFMD in China}

To obtain molecular epidemiological data on HFMD in China, we first searched all HFMD-related papers published both in English and Chinese between 1st January 2008 and 31st May 2018 using the keywords of "Prevalence" or "Detection" or "Outbreak" or "Epidemiology" or "Epidemic" or "Epidemic characteristics" or "Evolution", and "Hand food and mouth disease" or "HFMD" or "Enterovirus". The search for English papers was performed in the PubMed Database and the Web of Science (including conference proceedings). The Chinese literatures were searched from China National Knowledge Infrastructure (CNKI) and WanFang Database. In order to cover all regions of China, we searched publications from each province of China separately (including Hong Kong, Macao and Taiwan). If no paper was found in a province or municipality, further search was performed by cities in the province in order of the capital and the second largest city, or the names of the districts of the municipality. Only papers reporting the molecular epidemiological data on HFMD were kept. Because China (CDC) is responsible for the management and running of the national surveillance system for HFMD, to avoid possible data duplication, only data published by China CDC and local CDCs were used. If the data were reported by two or more papers, only those described in the most recent paper were used. As a result, a total of 64 publications, including 56 Chinese papers, and 8 English papers, were selected to decipher the molecular epidemiological trend of HFMD in China (Supplementary Table S1).

To analyze the national epidemiology of HFMD-related enteroviruses, we separated China into seven large regions: Northeast (Heilongjiang, Jilin, Liaoning), North (Inner Mongolia, Beijing, Tianjin, Hebei, Shanxi), East (Shandong, Jiangsu, Anhui, Zhejiang, Jiangxi, Fujian, Taiwan, Shanghai), Central (Henan, Hubei, Hunan), South (Guangdong, Guangxi, Hainan, Hongkong, Macao), Southwest (Sichuan, Guizhou, Yunnan, Chongqing, Tibet), Northwest (Xinjiang, Gansu, Qinghai, Shannxi, Ningxia) based on the Chinese Geographical Division (Fig. 1). The epidemiological data were analyzed by each region.

\section{Sequence Data of Enteroviruses EVA71, CVA16, CVA6 and CVA10 from China}

To investigate the evolution of the four main enteroviruses EVA71, CVA16, CVA6 and CVA10, all available partial VP1 sequences (Location: 2570-2872 nt in EVA71 Brcr 
strain, accession number U05876) of enteroviruses identified in China were retrieved from GenBank database by 31st June 2018. The sequences were grouped into different sets by provinces. After discarding erroneously annotated sequences that share less than $60 \%$ identity with reference sequences, and short sequences that overlap less than $270 \mathrm{nt}$ with the partial VP1 fragment, a total of 12,080 sequences were obtained. The sequences of each enterovirus were aligned with reference sequences described in previous studies (He et al. 2013; Zhang et al. 2015; Song et al. 2017; Ji et al. 2018). The sequence alignments were adjusted manually if needed, and subjected to the construction of maximum likelihood (ML) trees. To minimize the calculation during the ML tree construction, only one representative sequence was kept if there were two or more sequences sharing more than $97 \%$ genetic similarity (i.e. with a genetic distance of less than 3\%). The number of high-similarity sequences was marked following the name of each representative sequence. The removal of redundant sequences was performed using Python scripts. The ML trees were constructed using MEGA7.0 with 1000 bootstrap replications, and each enterovirus was genotyped according to previous studies (He et al. 2013; Zhang et al. 2015; Song et al. 2017; Ji et al. 2018). Enterovirus lineage was defined as forming a monophyletic clade in the ML tree and including over $2 \%$ of all sequences.

To compare the amino acid characteristics between lineages or genotypes of each enterovirus, the consensus amino acid sequences were displayed using WebLogo online Tool (http://weblogo.berkeley.edu/).

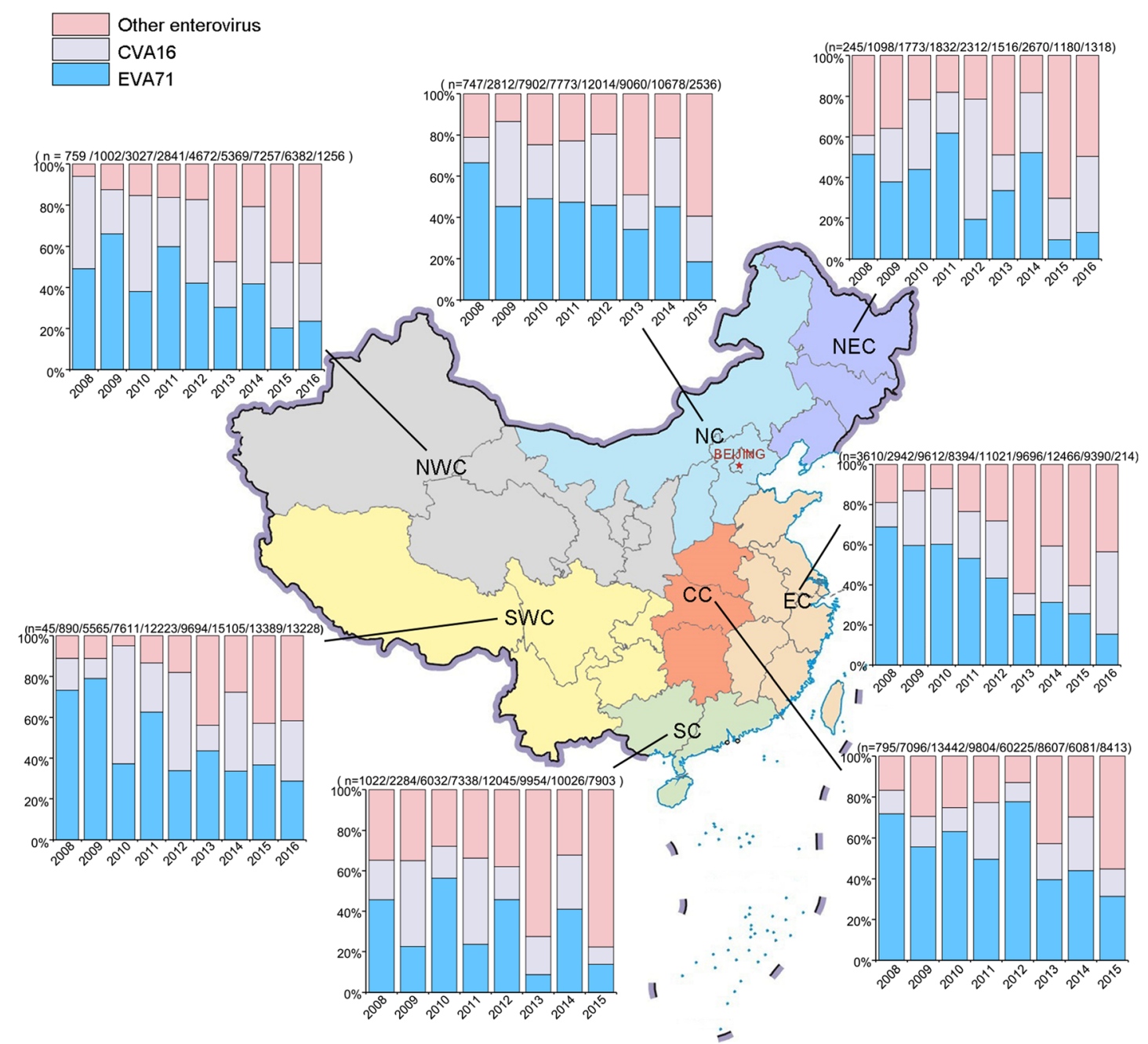

Fig. 1 Geographic distributions of EVA71, CVA16 and other enteroviruses in China from 2008 to 2016. The case number in each year is shown above each column. NEC, Northeast China; NWC,
Northwest China; NC, North China; CC, Central China; SC, South China; SWC, Southwest China; EC, East China. 


\section{Results}

\section{National Epidemiological Trend of Enteroviruses Associated with HFMD in China from 2008 to 2016}

China is administratively divided into seven large geographic regions, North, Northeast, Northwest, Central, East, Southern, and Southwest China. To decipher the national epidemiological trend of enteroviruses involved in HFMD, we analyzed the distributions of EVA71, CVA16 and non-EVA71 and non-CVA16 enteroviruses in the seven regions using data collected from 2008 to 2016. All regions showed a consistent trend of enterovirus prevalence (Fig. 1), with EVA71 and CVA16 being the top two enteroviruses responsible for HFMD in the early epidemics. A sharp decrease of EVA71 prevalence was observed in China from 2008 to 2016, accompanied by a relatively stable prevalence of CVA16. The decrease appeared to start from 2008 to 2009, with variation among regions. Notably, there was a rapid increase in the prevalence of enteroviruses other than EVA71 and CVA16 during the same period (Fig. 1), exceeding $50 \%$ of all genotyped enteroviruses (i.e. exceeded EVA71 and CVA 16 combined) in South, East and Northeast China in recent years, and implying that enteroviruses other than EVA71 and CVA16 have become the predominant agents of HFMD.

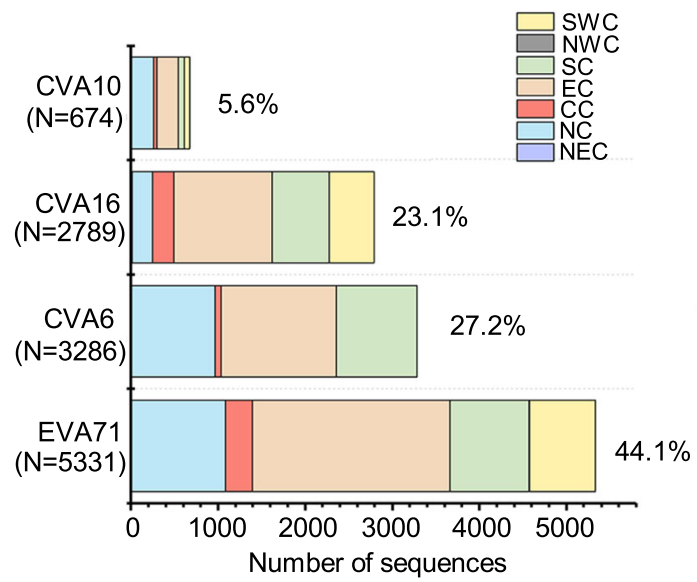

Fig. 2 Number of available partial $V P 1$ sequences of four main enteroviruses in China. A total of 12,080 sequences were obtained from GeneBank database by 31 June 2018. NEC, Northeast China; NWC, Northwest China; NC, North China; CC, Central China; SC, South China; SWC, Southwest China; EC, East China.

\section{Partial VP1 Sequences of Four Main HFMD Enteroviruses in China}

EVA71, CVA16, CVA6 and CVA10 are the four main enteroviruses that cause HFMD in China. In the GenBank database, a total of 12,080 partial $V P 1$ sequences of these four enteroviruses were from China by June 2018. Among them, EVA71 sequences accounted for $44.1 \%$, more than CVA16 and other serotypes, representing the predominant enterovirus for HFMD in the last decade in China (Fig. 2) (Liu et al. 2015; Li Y et al. 2018). Interestingly, the proportion of CVA6 sequences was $27.2 \%$, higher than that of CVA16 sequences $(23.1 \%)$, reflecting the rapid rise of CVA6 prevalence recently. CVA10 has the least abundance of $5.6 \%$ sequences.

Of note, the vast majority of the partial $V P I$ sequences were submitted from East China (e.g. Shanghai, Jiangsu, and Fujian), North China (e.g. Beijing) and South China (e.g. Guangdong), all of which are relatively developed areas. Only a few sequences were submitted from relatively undeveloped regions (e.g. Northwest and Northeast regions), implying more concerns should be focused on these regions.

\section{Evolutions of EVA71, CVA16, CVA6 and CVA10 in China}

To reveal the evolutionary relationship of EVA71 strains circulating in China, we re-constructed the phylogenetic tree using 179 representative partial VP1 sequences from 5331 EVA71 sequences in China (Fig. 3). All EVA71 genotypes $\mathrm{A}, \mathrm{B}$ and $\mathrm{C}$ were found in China. Genotype $\mathrm{C}$ contains five sub-genotypes $\mathrm{C} 1-\mathrm{C} 5$. Although the strains of $\mathrm{C} 1-\mathrm{C} 3$ and C5 were also found, vast majority (98.4\%) of the EVA71 strains belonged to $\mathrm{C} 4$ sub-genotype, representing the most predominant strain (Fig. 3). Among the C4 sub-genotype, three lineages $\mathrm{C} 4.1-\mathrm{C} 4.3$ were identified. Lineage EVA71_C4.1 has a larger proportion (65.9\%), with a longer epidemic period (2003-2017) and a broader geographic distribution than other lineages. Furthermore, a majority of the EVA71_C4.1 strains appeared to be circulating in East, North, Central and Southwest regions. Lineage EVA71_C4.2 accounted for $19.2 \%$, and it was mainly prevalent in North and South China, whereas lineage EVA71_C4.3 had a relatively short epidemic in the South regions.

The ML tree of CVA16 including 147 representative partial VP1 sequences from 2789 strains in China was shown in Fig. 4. A vast majority of the CVA16 strains belonged to B1 sub-genotype $(98.3 \%)$, representing the most predominant strain. Two B1 lineages (B1.1 and B1.2) were identified. Lineage CVA16_B1.1 was the most predominant strain, accounting for $63.4 \%$ of all B1 strains, and widely distributed nationally (Fig. 4). The majority of 


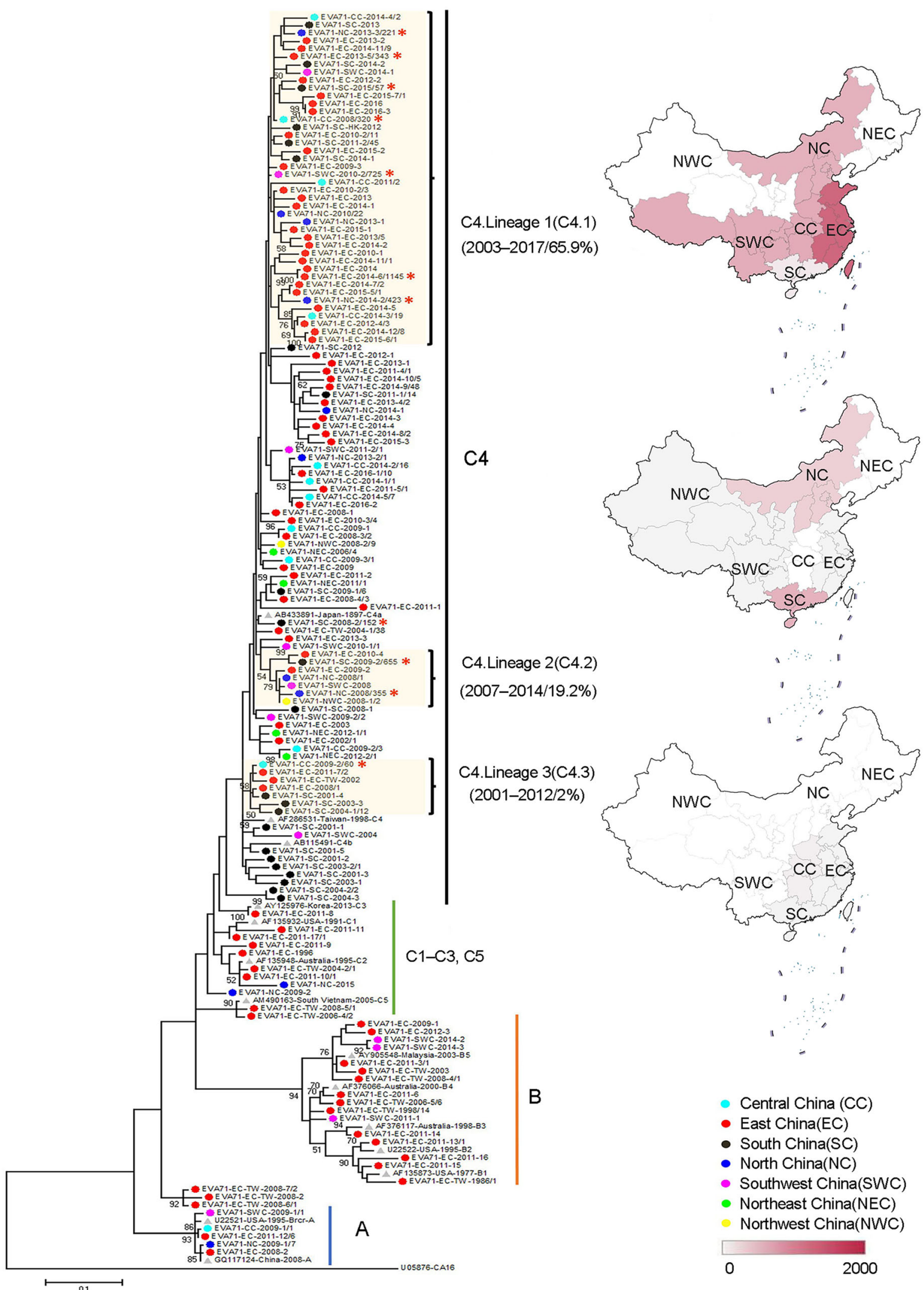

Fig. 3 Phylogenetic relationship of EVA71 strains using 179 representative partial VP1 sequences from 5331 EVA71 sequences from China. The representative sequences for more than 50 sequences sharing $97 \%$ sequence similarity are highlighted by red stars. Geographic distributions of lineages $\mathrm{C} 4.1, \mathrm{C} 4.2$ and $\mathrm{C} 4.3$ are shown on the right side (from top to bottom). 


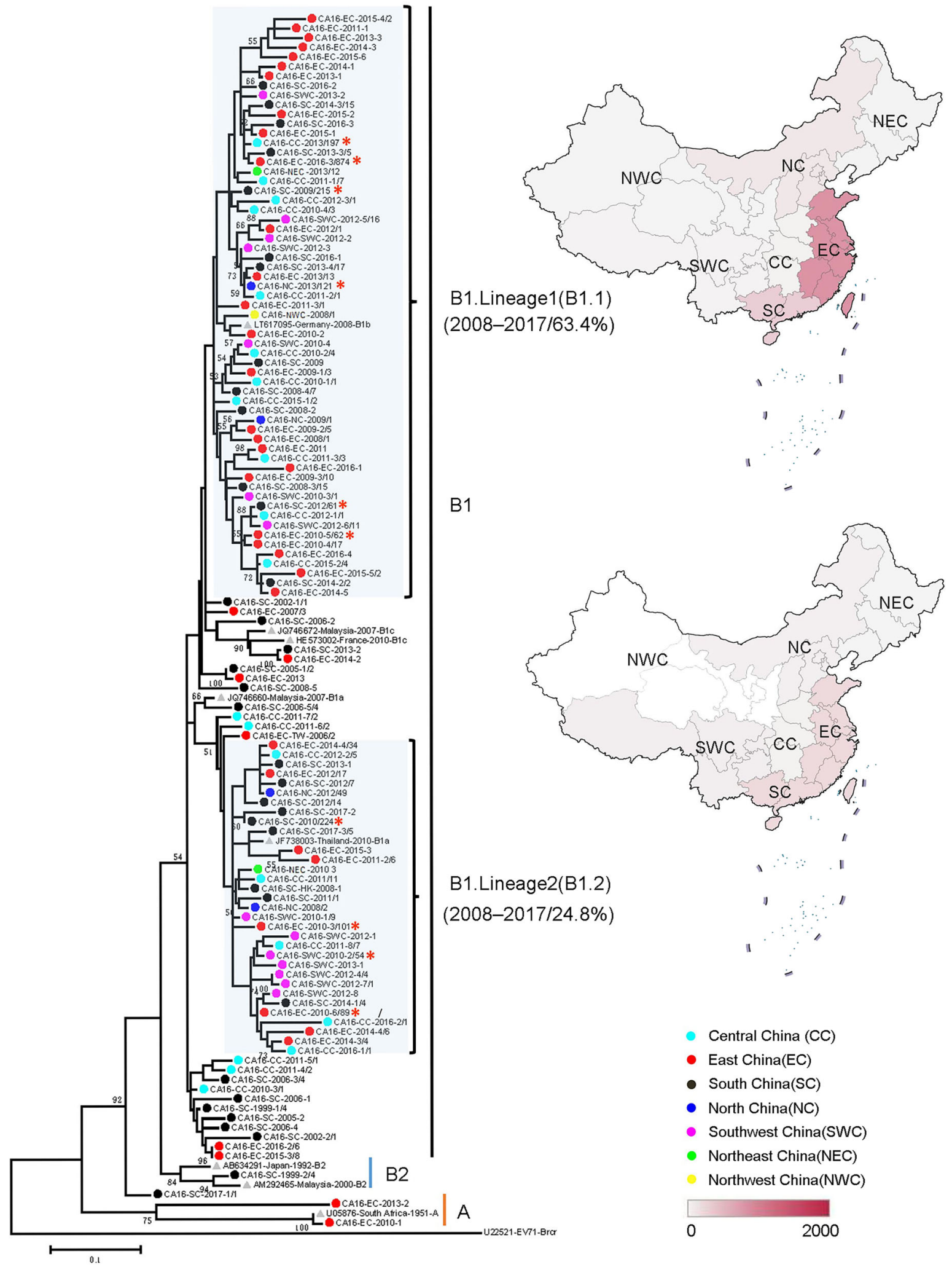

Fig. 4 Phylogenetic relationship of CVA16 strains using 147 representative partial VP1 sequences from 2789 strains from China. The representative sequences for more than 50 sequences sharing $97 \%$ sequence similarity are highlighted by red stars. Geographic distributions of lineages B1.1 and B1.2 are shown on the right side (from top to bottom). 
CVA16_B1.1 strains appeared to be circulating in East, South and North China. Lineage CVA16_B1.2 accounted for about one quarter $(24.8 \%)$, and it mainly circulated in East, South, North and Southwest China (Fig. 4).
The ML tree of CVA6 including 68 representative partial VPl sequences from 3286 strains in China was shown in Fig. 5. A vast majority of the CVA6 strains belonged to D genotype $(95.6 \%)$. As the most predominant strain,

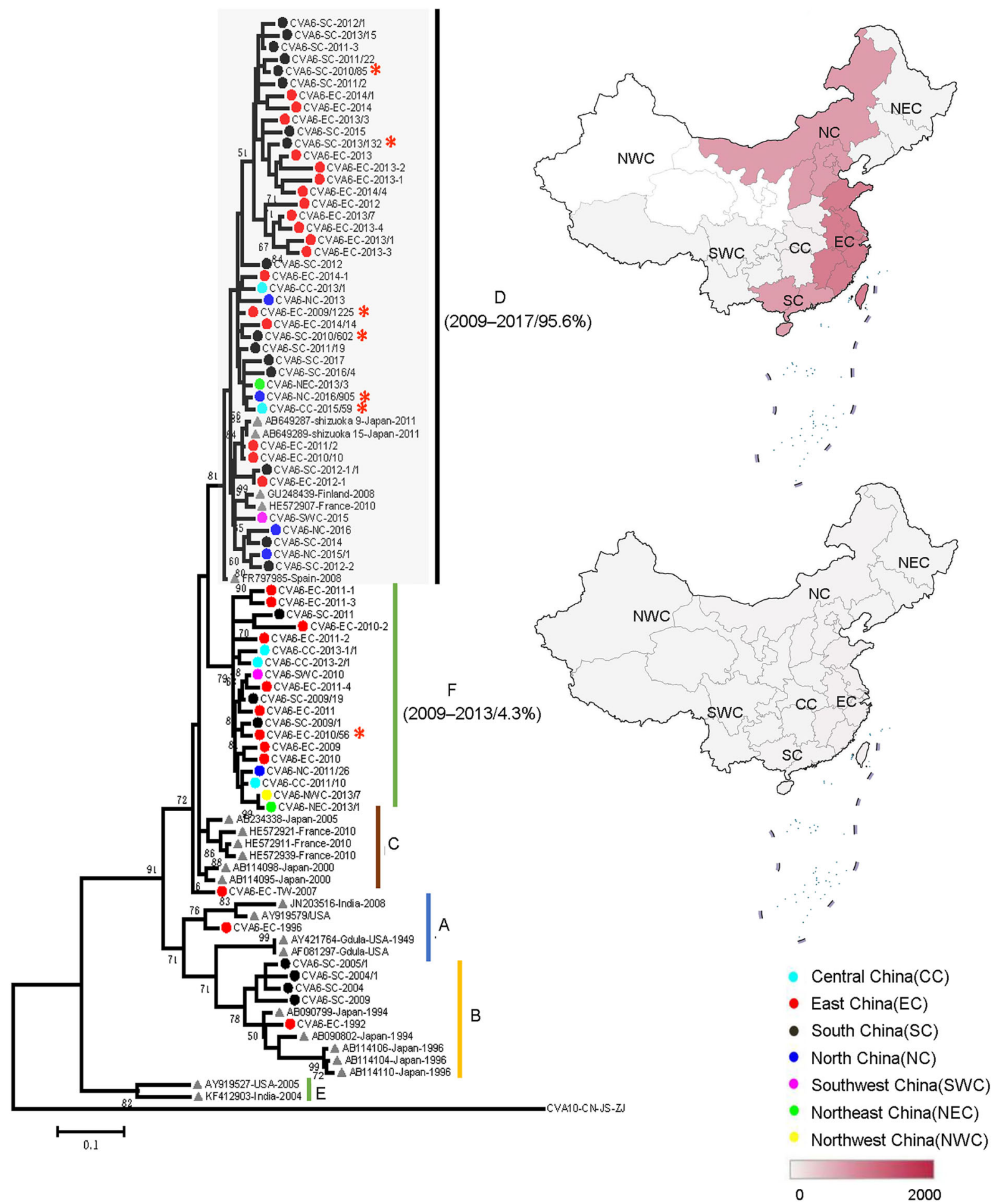

Fig. 5 Phylogenetic relationship of CVA6 strains including 68 representative partial VP1 sequences from 3286 strains from China. The representative sequences for more than 50 sequences sharing
97\% sequence similarity are highlighted by red stars. Geographic distributions of genotypes $\mathrm{D}$ and $\mathrm{F}$ are shown on the right side (from top to bottom). 
CVA6_D strain was mainly circulating in East, North and South regions (Fig. 5). CVA6_F genotype accounted for about $4.3 \%$ of CVA6 strains, and it was scattered in all seven regions of China, indicating a sporadic epidemic.

The ML tree of CVA10 including 47 representative partial VPl sequences from 674 strains in China was shown in Fig. 6. A vast majority of the CVA10 strains were C sub-genotype (97.3\%), representing the most predominant strains. The CVA10_C genotype first appeared in 2009, circulating mainly in East China. Interestingly, before 2009, CVA10_F genotype was the main CVA10 strain circulating in the South China, accounting for $2.7 \%$ of all sequences (Fig. 6).

As shown in the phylogenic trees (Figs. 3, 4, 5, 6), different genotypes of the same enterovirus have very short genetic distance. Further comparison of amino acid sequences of the partial VPl genes revealed no significant difference among these main genotypes, except for CVA10 that has three amino acid differences between genotypes $\mathrm{C}$ and F (Supplementary Fig. S1).

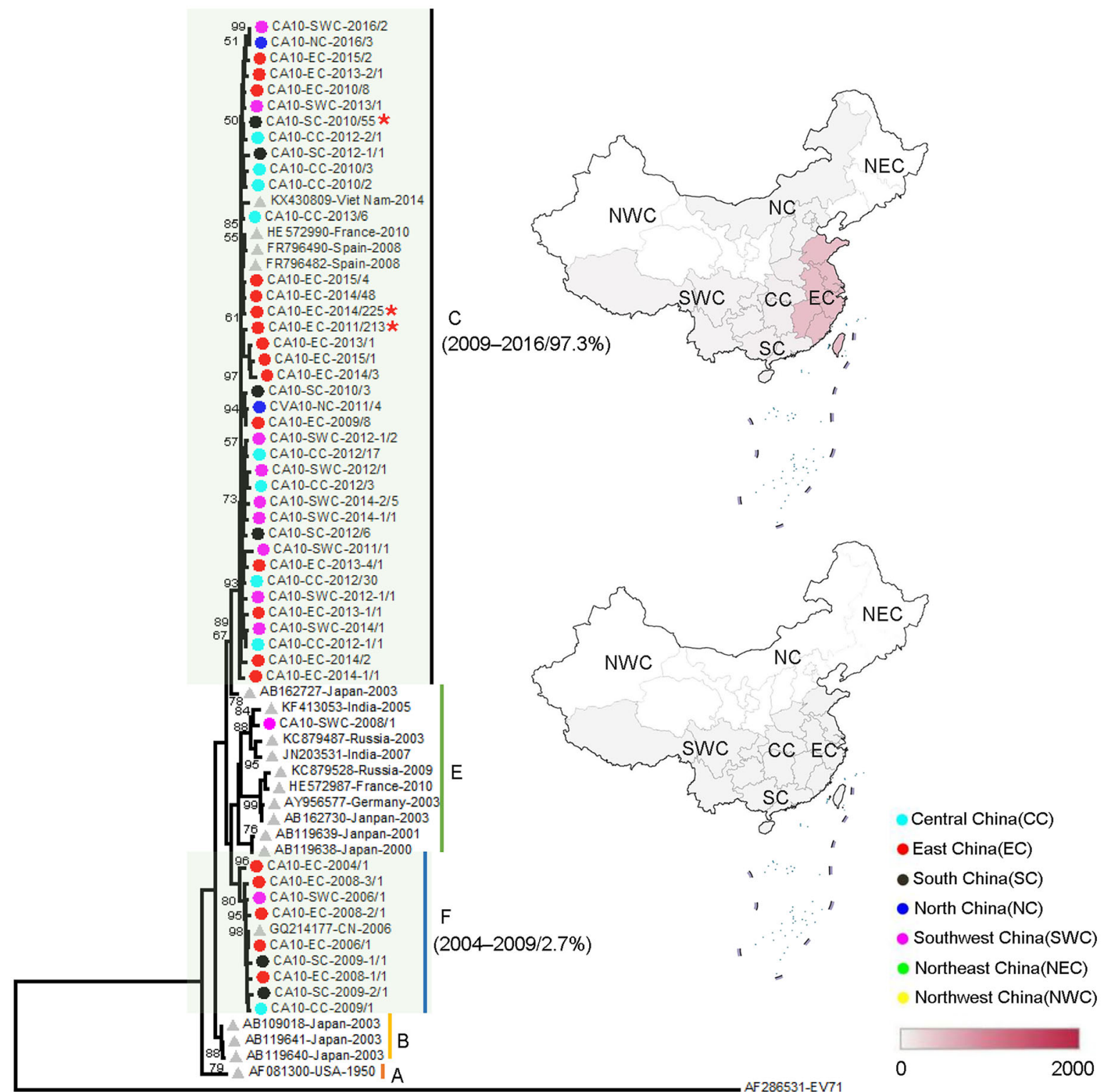

ㅂ.1ㄴ.

Fig. 6 Phylogenetic relationship of CVA10 strains including 47 representative partial $V P 1$ sequences from 674 strains from China. The representative sequences for more than 50 sequences sharing
97\% sequence similarity are highlighted by red stars. Geographic distributions of genotypes $\mathrm{C}$ and $\mathrm{F}$ are shown on the right side (from top to bottom). 
Fig. 7 Timeline and cocirculation of several main enterovirus lineages in China in 2001-2017. A Timeline. The time does not represent the origin of the lineage, which needs to be determined by Bayesian evolutionary analysis. B Geographic profile of the main co-circulated enterovirus lineages described in

A. Because few sequences were obtained from Northeast and Northwest China, to avoid bias, the data of these two regions are not shown.
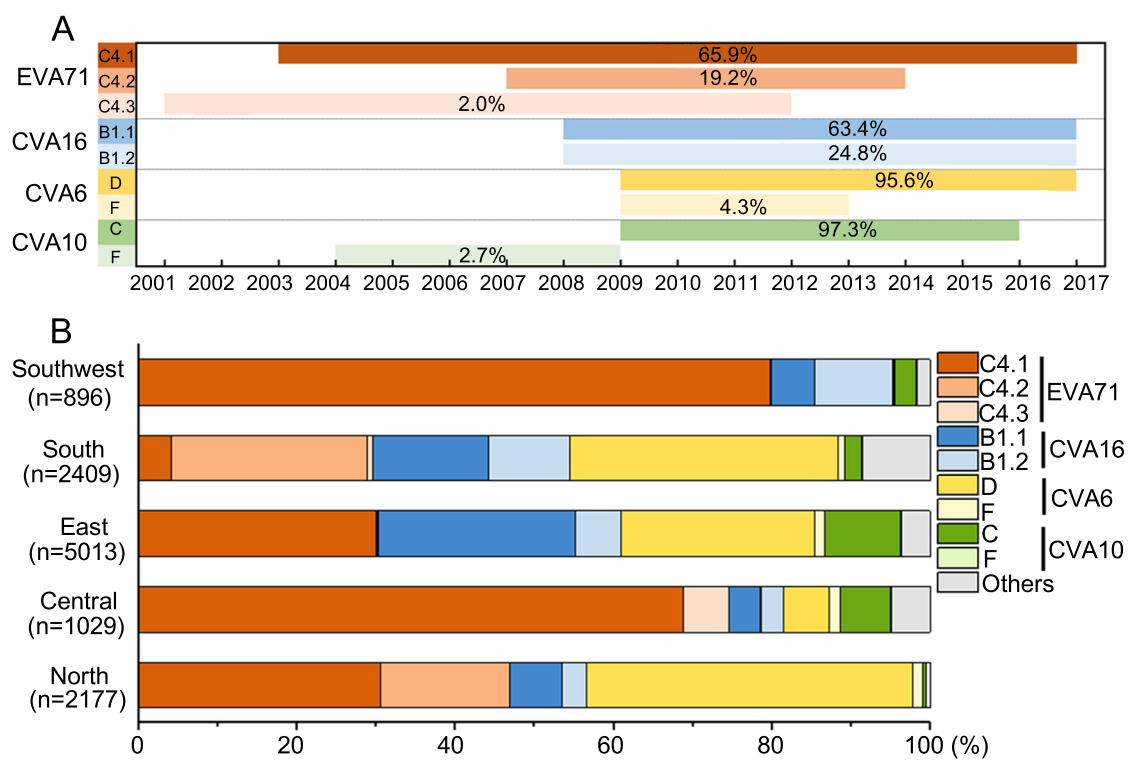

\section{Co-circulation of Several Main Enterovirus Lineages or Genotypes}

Based on phylogenic analyses of EV71, CA16, CA10 and CA6, three EVA71_C4 lineages, two CVA16_B1 lineages, two CVA6 genotypes and two CVA10 genotypes were identified as the major viral strains that cause HFMD epidemics in China (Figs. 3, 4, 5, 6). For each enterovirus, there was one dominant lineage or genotype in a given HFMD epidemic (Fig. 7A). Timeline analysis revealed that EVA71 has a longer epidemic period, and started to cocirculate with the other lineages or genotypes of enteroviruses (i.e., CVA16, CVA6 and CVA10) since 2009 (Fig. 7A). Further co-circulation analyses showed that lineages and genotypes of the co-circulated enteroviruses varied largely in different regions of China (Fig. 7B). In Central and Southwest China, lineage EVA71-C4.1 was the most predominant enterovirus co-circulating with other enteroviruses. Of note, there were three to four predominant enterovirus lineages or genotypes co-circulating in North, East, and South China with similar prevalence rates (Fig. 7B). The top three lineages/genotypes were CVA6_D (41.4\%), EVA71_C4.1 (30.7\%), and EVA71_C4.2 (16.4\%) in North China, EVA71_C4.1 (31.2\%), CVA16_B1.1 (25.9\%), and CVA6_D (25.5\%) in East China, and CVA6_D (37.1\%), EVA71_C4.2 (27.1\%), and CVA16_B1.1 (16.1\%) in South China.

\section{Discussion}

A shift in the molecular epidemiology of enteroviruses affects policy-making for the prevention and control of HFMD in China. In this study, we present a meta-analysis demonstrating a rapid increase in the prevalence of enteroviruses other than EVA71 and CVA16 from 2008 to 2016, accompanied with a sharp decrease of EVA71 prevalence in China (Fig. 1). These findings have several significant implications on the virological surveillance of HFMD, pathogenesis of enteroviruses and HFMD vaccine development.

First, the overall trend in molecular epidemiology of HFMD-related enteroviruses suggests that more virological surveillance efforts should be focused on the enteroviruses other than EVA71 and CVA16 in the future ( $\mathrm{Li} \mathrm{Y}$ et al. 2018). During the early phase of HFMD epidemic in China, the majority of the cases were caused by EVA71 and CVA16. To identify the causative agents of HFMD, especially for severe cases, it is mandatory to test for EVA71 and CVA16 infection using specific RT-qPCR assays or serological tests accordingly to the existing national virological surveillance guideline. Although the detection of enteroviruses other than EVA71 and CVA16 by a universal RT-qPCR assay was also performed in a few regions, certain serotypes cannot be distinguished by specific RT-qPCR assays or Sanger sequencing (Xing et al. 2014). In view of the fact that CVA6 and CVA10 had a rapidly increasing prevalence in recent years (Fig. 1), a pan-enterovirus detection kit with universal primer sets has recently been developed (Shen et al. 2018; Wang M et al. 2018). Our results support its deployment.

Second, more molecular epidemiological efforts should be focused on enteroviruses other than EVA71 and CVA16, especially in undeveloped regions of China. Compared to EVA71, far fewer partial VPI sequences were available from CVA16 and other enteroviruses (Fig. 2), limiting the capacity for a better understanding of the evolution of enteroviruses other than EVA71. Furthermore, 
according to geographic distribution, a vast majority of the sequences were from the developed regions (e.g. Beijing, Shanghai, Guangdong, Jiangsu and Fujian), whereas far fewer sequences were from the undeveloped regions, especially from the national border provinces. HFMD is a serious public health threat not only to China, but also to other Asia countries (Van Tu et al. 2007; Khanh et al. 2012; Nguyen et al. 2014; Sabanathan et al. 2014; Duong et al. 2016; Kim et al. 2016; Koh et al. 2016; NikNadia et al. 2016). In particular, some large HFMD outbreaks occurred in recent years in the neighboring countries including Korea, Russia, Vietnam, and Cambodia. Moreover, a few studies have suggested a cross-border transmission of enteroviruses among Southeast Asia countries (Tee et al. 2010; Geoghegan et al. 2015). To better clarify the cross-border transmission of enteroviruses, more viral sequences from these national border provinces (e.g. Yunnan, Guangxi, Liaoning, etc.) will be very helpful.

In addition to the partial gene sequences, full-length genomic sequences can provide more information on the genetic determinants of viral biological traits (virulence, fitness, pathogenicity, etc.), and the evolutionary history (origin, transmission, and recombination events, etc.) of certain enterovirus (Simmonds and Welch 2006; Zhang et al. 2013; Geoghegan et al. 2015). A vast majority of severe (60\%) and fatal (90\%) cases of HFMD was caused by EVA71 (Liu et al. 2015); however, no significant difference was observed in the EVA71 genomic sequences between the mild and severe HFMD cases (Zhang et al. 2013), suggesting that the genomic characteristics of EVA71 was not the main determinant of disease severity. Apart from EVA71, about 9\% fatal cases were associated with the enteroviruses other than EVA71 and CVA16 (Liu et al. 2015). Which enterovirus causes deaths and whether it carries special genetic traits are important questions for further molecular epidemiological investigation and comparative genomic analysis. In recent years, the decreasing cost of next generation sequencing (NGS) makes it possible to easily obtain viral genomic sequences from clinical samples (Geoghegan et al. 2015). Genomic sequencing of enteroviruses (especially for those that are not EVA71 and CVA16) using NGS is also especially encouraged.

Third, monovalent or multivalent vaccine against enteroviruses other than EVA71 (e.g. CVA6, CVA16, and CVA10) should be developed. Vaccination is the most effective way to protect younger children from HFMD in the absence of an effective antiviral drug. Currently, only three highly efficacious monovalent EVA71 vaccines were licensed in China in late 2015, and these EVA71 vaccines did not show cross-protection against other serotypes including CVA16 (Zhu et al. 2013; Li R et al. 2014). The sharply decreasing prevalence of EVA71 and rapidly increasing contribution of enteroviruses other than EVA71 and CVA16 to HFMD urgently call for development of monovalent or multivalent vaccine against CVA6, CVA16, and CVA10 in addition to EVA71. It is interesting to note that EVA71 prevalence decreased gradually from 2009 to 2016, before the wide deployment and licensure of EVA71 vaccines. Reduced viral diversity was previously demonstrated to lead to lower viral virulence and fitness (Furio et al. 2005). Compared to CVA6, CVA16, and CVA10, EVA71 appears to have a lower mutation rate (Lukashev and Vakulenko 2017). A relatively lower viral diversity of EVA71 might reduce its fitness and transmissibility, which provides a potential explanation for the decrease of EVA71 prevalence. In contrast, higher viral diversity may contribute to the increase of CVA6 prevalence (Lukashev and Vakulenko 2017; Anh et al. 2018).

RNA viruses have high mutation rate. Another important finding of this study is that one genotype of each enterovirus contributed to the main HFMD epidemic in a given geographic location (Figs. 3, 4, 5, 6, 7). Each enterovirus was classified into different genotypes or subgenotypes. Sub-genotypes EVA71_C4, CVA16_B1, CVA6_D and CVA10_C were found to contribute to over $90 \%$ of all HFMD cases caused by the four serotypes. This information is beneficial to the HFMD vaccine development. In addition, we identified three lineages in EVA71 sub-genotype $\mathrm{C} 4$ and two lineages in CVA16 sub-genotype B1. The lineages EVA71_C4.1 and CVA16_B1.1 contributed to $65.9 \%$ and $63.4 \%$ of the HFMD cases caused by the corresponding enterovirus, respectively (Figs. 3, 4). RNA viruses in general exist as quasispecies, and some variants with specific genetic changes in their genome can alter their virulence, transmissibility and pathogenicity (Sanz-Ramos et al. 2008; Anh et al. 2018). To address whether the predominant lineages were associated with some special genetic changes, we compared the consensus amino acid sequences between different lineages. No significant difference was observed for EVA71, CVA16 and CVA6 (Supplementary Fig. S1), giving no support to the hypotheses that greater fitness associates with specific lineage, but implying the results represent simply a founder's effect. Whether herd immunity in the population drives the evolution of various enterovirus lineages needs further investigation. Interestingly, we found there were three amino acid differences between CVA10 genotypes C and $\mathrm{F}$. Whether the three amino acid changes were the genetic determinants for the predominance of genotype $\mathrm{C}$ requires further experimental investigation. Furthermore, because only partial VPl sequences were analyzed, changes in other genomic regions need to be determined in the future.

A distinct geographic distribution was observed for EVA71, CVA16, CVA10 and CVA6 (Fig. 7); however, the distribution was not obviously different between South and 
North China, despite there were two seasonal peaks in South regions but only a single-peak in North regions (Xing et al. 2014; Mao et al. 2018). Higher proportions of EVA71_C4.1 were found in Southwest $(81.1 \%)$ and Central $(72.4 \%)$ China, where a few other enterovirus strains co-circulated. In contrast, higher proportions of CVA16_B1.1 and CVA6_D strains were observed in South, East and North China. Importantly, co-circulation of four enteroviruses with similar proportions in South, East and North China might be associated with higher population density of children and contributed to higher HFMD incidence in these areas (Huang et al. 2014; Wang et al. 2016). On the other hand, the co-circulation of these enteroviruses also provided a ready opportunity for recombination between different enteroviruses (Simmonds and Welch 2006). Indeed, some recombinants of enteroviruses had been identified in East and South China (e.g. Shandong, Jiangsu, Shanghai and Taiwan) (Hu et al. 2011; Zhang et al. 2015). Apart from the four main serotypes, some rare serotypes were also co-circulating with the four main enteroviruses in some regions (Lu et al. 2012; Guan et al. 2015; Li et al. 2017), suggesting the possibility of generation of new recombinants. Recombination plays a crucial role in the evolution of enterovirus (Simmonds and Welch 2006). The hot spots for enterovirus recombination are often located in the 2A/2B genomic regions (Nikolaidis et al. 2018). Because the analyzed sequences are predominantly represented by partial VPl genomic region, some circulating enterovirus recombinants may be missed by this and other studies. To find potential recombinants, fulllength genomic sequence analyses are needed. In areas where scientific and fiscal resources are limited, an alternative simple strategy of amplifying and sequencing the $2 \mathrm{~A} / 2 \mathrm{~B}$ genomic region is strongly encouraged.

This study has two limitations. First, there were very limited sequences available from some undeveloped areas in China and thus the presentation of spatial and temporal distribution in these regions may not be precise. Second, because full-length genomic sequences (including untranslated regions) that offer more comprehensive information on genetic and evolution of enteroviruses are mostly not available, we only studied VPI sequences. Given the findings and limitations of this study, future studies should be focused on CVA16 and other enteroviruses especially in some undeveloped areas with fulllength genomic sequencing. Furthermore, comparative analyses of sequences and phylogenetic relationships between enteroviruses from China and global strains, and seroepidemiologic investigations on the correlation between the circulating enterovirus strains and the prevalence of the relevant enterovirus-specific antibodies in the population should also be encouraged.
In summary, we report a rapid increase of other enteroviruses in accompany with a sharp decrease of EVA71 prevalence in China from 2008 to 2016. In addition, we find that four genotypes EVA71_C4, CVA16_B1, CVA6_D and CVA10_C contributed to over 90\% of all HFMD cases in China. The epidemiological and evolutionary information of the four main enteroviruses have significant implications for virological surveillance, disease management and vaccine development, and provide scientific basis for policy-making on the prevention and control of HFMD.

Acknowledgements We thank Zhihang Zheng and Rong $\mathrm{Xu}$ at Institut Pasteur of Shanghai, CAS for their technique help in this study. This work was supported by the TOTAL foundation, and the Grants from the National Science and Technology Major Project of China (2017ZX10103009-002), the "One Belt One Road" Project (153831KYSB20170043) of the Chinese Academy of Sciences, the 133 Project of Institut Pasteur of Shanghai, CAS.

Author Contributions $\mathrm{CZ}$ conceived and designed the study. XF, $\mathrm{ZW}, \mathrm{XJ}$ and $\mathrm{YH}$ collected the data. XF, ZW, and YL performed the analyses. $\mathrm{CZ}, \mathrm{XF}$ and $\mathrm{ZW}$ analyzed and interpreted the data. $\mathrm{XF}$ and $\mathrm{CZ}$ drafted the manuscript, and $\mathrm{XJ}$ contributed to critical revision of the manuscript. All authors have read and approved the contents of the final manuscript.

\section{Compliance with Ethical Standards}

Animal and Human Rights Statement This article does not contain any studies with human or animal subjects performed by any of the authors.

\section{References}

Anh NT, Nhu LNT, Van HMT, Hong NTT, Thanh TT, Hang VTT, Ny NTH, Nguyet LA, Phuong TTL, Nhan LNT, Hung NT, Khanh TH, Tuan HM, Viet HL, Nam NT, Viet DC, Qui PT, Wills B, Sabanathan S, Chau NVV, Thwaites L, Rogier van Doorn H, Thwaites G, Rabaa MA, Van Tan L (2018) Emerging coxsackievirus A6 causing hand, foot and mouth disease, Vietnam. Emerg Infect Dis 24:654-662

Bian L, Wang Y, Yao X, Mao Q, Xu M, Liang Z (2015) Coxsackievirus A6: a new emerging pathogen causing hand, foot and mouth disease outbreaks worldwide. Expert Rev Anti Infect Ther 13:1061-1071

De W, Changwen K, Wei L, Monagin C, Jin Y, Cong M, Hanri Z, Jun S (2011) A large outbreak of hand, foot, and mouth disease caused by EV71 and CAV16 in Guangdong, China, 2009. Arch Virol 156:945-953

Duong V, Mey C, Eloit M, Zhu H, Danet L, Huang Z, Zou G, Tarantola A, Cheval J, Perot P, Laurent D, Richner B, Ky S, Heng S, Touch S, Sovann L, van Doorn R, Tan Tran T, Farrar JJ, Wentworth DE, Das SR, Stockwell TB, Manuguerra JC, Delpeyroux F, Guan Y, Altmeyer R, Buchy P (2016) Molecular epidemiology of human enterovirus 71 at the origin of an epidemic of fatal hand, foot and mouth disease cases in Cambodia. Emerg Microb Infect 5:e104 
Fu X, Mao L, Wan Z, Xu R, Ma Y, Shen L, Jin X, Zhang C (2019) High proportion of coxsackievirus B3 genotype A in hand, foot and mouth disease in Zhenjiang, China, 2011-2016. Int J Infect Dis $87: 1-7$

Furio V, Moya A, Sanjuan R (2005) The cost of replication fidelity in an RNA virus. Proc Natl Acad Sci USA 102:10233-10237

Geoghegan JL, Kühnert D, Halpin RA, Lin X, Simenauer A, Akopov A, Das SR, Stockwell TB, Shrivastava S, Ngoc NM (2015) Phylodynamics of enterovirus A71-associated hand, foot and mouth disease in Vietnam. J Virol 89:8871-8879

Guan H, Wang J, Wang C, Yang M, Liu L, Yang G, Ma X (2015) Etiology of multiple non-EV71 and non-CVA16 enteroviruses associated with hand, foot and mouth disease in Jinan, China, 2009-June 2013. PLoS One 10:e142733

He Y-Q, Chen L, Xu W-B, Yang H, Wang H-Z, Zong W-P, Xian H-X, Chen H-L, Yao X-J, Hu Z-L (2013) Emergence, circulation and spatiotemporal phylogenetic analysis of coxsackievirus A6 and coxsackievirus A10 associated hand, foot and mouth disease infections from 2008 to 2012 in Shenzhen, China. J Clin Microbiol 51:3560-3566

Hu Y, Yang F, Du J, Dong J, Zhang T, Wu Z, Xue Y, Jin Q (2011) Complete genome analysis of coxsackievirus A2, A4, A5, and A10 strains isolated from hand-foot-and-mouth disease patients in China revealing frequent recombination of human enterovirus A. J Clin Microbiol 49:2426-2434

Huang J, Wang J, Bo Y, Xu C, Hu M, Huang D (2014) Identification of health risks of hand, foot and mouth disease in China using the geographical detector technique. Int J Environ Res Public Health 11:3407-3423

Ji T, Guo Y, Huang W, Shi Y, Xu Y, Tong W, Yao W, Tan Z, Zeng $\mathrm{H}$, Ma J (2018) The emerging sub-genotype C2 of coxsackievirus A10 associated with hand, foot and mouth disease extensively circulating in Mainland of China. Sci Rep 8:13357

Khanh TH, Sabanathan S, Thanh TT, le Thoa PK, Thuong TC, Hang V, Farrar J, Hien TT, Chau N, van Doorn HR (2012) Enterovirus 71-associated hand, foot, and mouth disease, Southern Vietnam, 2011. Emerg Infect Dis 18:2002-2005

Kim HJ, Hyeon JY, Hwang S, Lee YP, Lee SW, Yoo JS, Kang B, Ahn JB, Jeong YS, Lee JW (2016) Epidemiology and virologic investigation of human enterovirus 71 infection in the Republic of Korea from 2007 to 2012: a nationwide cross-sectional study. BMC Infect Dis 16:425

Koh WM, Bogich T, Siegel K, Jin J, Chong EY, Tan CY, Chen MI, Horby P, Cook AR (2016) The epidemiology of hand, foot and mouth disease in Asia: a systematic review and analysis. Pediatr Infect Dis J 35:e285-e300

Li L, He Y, Yang H, Zhu J, Xu X, Dong J, Zhu Y, Jin Q (2005) Genetic characteristics of human enterovirus 71 and coxsackievirus A16 circulating from 1999 to 2004 in Shenzhen, People's Republic of China. J Clin Microbiol 43:3835-3839

Li J, Yuan J, Yang F, Wu Z-Q, Hu Y-F, Xue Y, Zhou B-P, Jin Q (2014) Epidemic characteristics of hand, foot, and mouth disease in southern China, 2013: coxsackievirus A6 has emerged as the predominant causative agent. J Infect 69:299-303

Li R, Liu L, Mo Z, Wang X, Xia J, Liang Z, Zhang Y, Li Y, Mao Q, Wang J (2014) An inactivated enterovirus 71 vaccine in healthy children. N Engl J Med 370:829-837

Li Y, Bao H, Zhang X, Zhai M, Bao X, Wang D, Zhang S (2017) Epidemiological and genetic analysis concerning the nonenterovirus 71 and non-coxsackievirus A16 causative agents related to hand, foot and mouth disease in Anyang city, Henan Province, China, from 2011 to 2015. J Med Virol 89:1749-1758

Li J, Pan H, Wang X, Zhu Q, Ge Y, Cai J, Li Y, Xia A, Hu J, Zeng M (2018) Epidemiological surveillance of hand, foot and mouth disease in Shanghai in 2014-2016, prior to the introduction of the enterovirus 71 vaccine. Emerg Microb Infect 7:37
Li Y, Chang Z, Wu P, Liao Q, Liu F, Zheng Y, Luo L, Zhou Y, Chen Q, Yu S (2018) Emerging enteroviruses causing hand, foot and mouth disease, China, 2010-2016. Emerg Infect Dis 24:1902

Liu W, Wu S, Xiong Y, Li T, Wen Z, Yan M, Qin K, Liu Y, Wu J (2014) Co-circulation and genomic recombination of coxsackievirus A16 and enterovirus 71 during a large outbreak of hand, foot, and mouth disease in central China. PLoS One 9:e96051

Liu SL, Pan H, Liu P, Amer S, Chan TC, Zhan J, Huo X, Liu Y, Teng Z, Wang L, Zhuang H (2015) Comparative epidemiology and virology of fatal and nonfatal cases of hand, foot and mouth disease in Mainland China from 2008 to 2014. Rev Med Virol 25:115-128

Lu Q-B, Zhang X-A, Wo Y, Xu H-M, Li X-J, Wang X-J, Ding S-J, Chen X-D, He C, Liu L-J (2012) Circulation of coxsackievirus A10 and A6 in hand-foot-mouth disease in China, 2009-2011. PLoS One 7:e52073

Lukashev AN, Vakulenko YA (2017) Molecular evolution of types in non-polio enteroviruses. J Gen Virol 98:2968-2981

Mao LX, Wu B, Bao WX, Han FA, Xu L, Ge QJ, Yang J, Yuan ZH, Miao CH, Huang XX, Zhang C, Xu H (2010) Epidemiology of hand, foot, and mouth disease and genotype characterization of enterovirus 71 in Jiangsu, China. J Clin Virol 49:100-104

Mao L, Fu X, Wu J, Shen L, Gu J, Yuan Z, Chen J, Zou X, Zhang C (2018) The dynamics of the hand, foot and mouth disease epidemic from 2008 to 2016 in Zhenjiang city, China. Future Microbiol 13:1029-1040

Nguyen NT, Pham HV, Hoang CQ, Nguyen TM, Nguyen LT, Phan HC, Phan LT, Vu LN, Tran Minh NN (2014) Epidemiological and clinical characteristics of children who died from hand, foot and mouth disease in Vietnam, 2011. BMC Infect Dis 14:341

NikNadia N, Sam IC, Rampal S, WanNorAmalina W, NurAtifah G, Verasahib K, Ong CC, MohdAdib M, Chan YF (2016) Cyclical patterns of hand, foot and mouth disease caused by enterovirus A71 in Malaysia. PLoS Negl Trop Dis 10:e0004562

Nikolaidis M, Mimouli K, Kyriakopoulou Z, Tsimpidis M, Tsakogiannis D, Markoulatos P, Amoutzias GD (2018) Large-scale genomic analysis reveals recurrent patterns of intertypic recombination in human enteroviruses. Virology 526:72-80

Ooi MH, Wong SC, Lewthwaite P, Cardosa MJ, Solomon T (2010) Clinical features, diagnosis, and management of enterovirus 71 . Lancet Neurol 9:1097-1105

Sabanathan S, le Tan V, Thwaites L, Wills B, Qui PT, Rogier van Doorn H (2014) Enterovirus 71 related severe hand, foot and mouth disease outbreaks in South-East Asia: current situation and ongoing challenges. $\mathrm{J}$ Epidemiol Community Health 68:500-502

Sanz-Ramos M, Diaz-San Segundo F, Escarmis C, Domingo E, Sevilla N (2008) Hidden virulence determinants in a viral quasispecies in vivo. J Virol 82:10465-10476

Shen XX, Qiu FZ, Zhao HL, Yang MJ, Hong L, Xu ST, Zhou SF, Li GX, Feng ZS, Ma XJ (2018) A novel and highly sensitive realtime nested RT-PCR assay in a single closed tube for detection of enterovirus. Diagn Microbiol Infect Dis 90:181-185

Simmonds P, Welch J (2006) Frequency and dynamics of recombination within different species of human enteroviruses. J Virol 80:483-493

Song Y, Zhang Y, Ji T, Gu X, Yang Q, Zhu S, Xu W, Xu Y, Shi Y, Huang X (2017) Persistent circulation of coxsackievirus A6 of genotype D3 in Mainland of China between 2008 and 2015. Sci Rep 7:5491

Tee KK, Lam TT, Chan YF, Bible JM, Kamarulzaman A, Tong CY, Takebe Y, Pybus OG (2010) Evolutionary genetics of human enterovirus 71: origin, population dynamics, natural selection, and seasonal periodicity of the VP1 gene. J Virol 84:3339-3350

Van Tu P, Thao NTT, Perera D, Truong KH, Tien NTK, Thuong TC, How OM, Cardosa MJ, McMinn PC (2007) Epidemiologic and 
virologic investigation of hand, foot, and mouth disease, southern Vietnam, 2005. Emerg Infect Dis 13:1733-1741

Vignuzzi M, Stone JK, Arnold JJ, Cameron CE, Andino R (2006) Quasispecies diversity determines pathogenesis through cooperative interactions in a viral population. Nature 439:344-348

Wang C, Li X, Zhang Y, Xu Q, Huang F, Cao K, Tao L, Guo J, Gao Q, Wang W, Fang L, Guo X (2016) Spatiotemporal cluster patterns of hand, foot, and mouth disease at the county level in Mainland China, 2008-2012. PLoS One 11:e147532

Wang J, Hu T, Sun D, Ding S, Carr MJ, Xing W, Li S, Wang X, Shi W (2017) Epidemiological characteristics of hand, foot, and mouth disease in Shandong, China, 2009-2016. Sci Rep 7:8900

Wang C, Zhou S, Xue W, Shen L, Huang W, Zhang Y, Li X, Wang J, Zhang H, Ma X (2018) Comprehensive virome analysis reveals the complexity and diversity of the viral spectrum in pediatric patients diagnosed with severe and mild hand-foot-and-mouth disease. Virology 518:116-125

Wang M, Ren Q, Zhang Z, Zhang L, Carr MJ, Li J, Zhou H, Shi W (2018) Rapid detection of hand, foot and mouth disease enterovirus genotypes by multiplex PCR. J Virol Methods 258:7-12

Xing W, Liao Q, Viboud C, Zhang J, Sun J, Wu JT, Chang Z, Liu F, Fang VJ, Zheng Y, Cowling BJ, Varma JK, Farrar JJ, Leung GM, Yu H (2014) Hand, foot, and mouth disease in China, 2008-12: an epidemiological study. Lancet Infect Dis $14: 308-318$
Xu M, Su L, Cao L, Zhong H, Dong N, Dong Z, Xu J (2015) Genotypes of the enterovirus causing hand foot and mouth disease in Shanghai, China, 2012-2013. PLoS One 10:e0138514

Yang B, Liu F, Liao Q, Wu P, Chang Z, Huang J, Long L, Luo L, Li Y, Leung GM (2017) Epidemiology of hand, foot and mouth disease in China, 2008 to 2015 prior to the introduction of EVA71 vaccine. Eurosurveillance 22

Zhang Y, Tan X, Cui A, Mao N, Xu S, Zhu Z, Zhou J, Shi J, Zhao Y, Wang X, Huang X, Zhu S, Zhang Y, Tang W, Ling H, Xu W (2013) Complete genome analysis of the C4 subgenotype strains of enterovirus 71: predominant recombination $\mathrm{C} 4$ viruses persistently circulating in China for 14 years. PLoS One 8:e56341

Zhang C, Zhu R, Yang Y, Chi Y, Yin J, Tang X, Yu L, Zhang C, Huang Z, Zhou D (2015) Phylogenetic analysis of the major causative agents of hand, foot and mouth disease in Suzhou City, Jiangsu province, China, in 2012-2013. Emerg Microb Infect 4:e12

Zhu F-C, Meng F-Y, Li J-X, Li X-L, Mao Q-Y, Tao H, Zhang Y-T, Yao X, Chu K, Chen Q-H (2013) Efficacy, safety, and immunology of an inactivated alum-adjuvant enterovirus 71 vaccine in children in China: a multicentre, randomised, doubleblind, placebo-controlled, phase 3 trial. Lancet 381:2024-2032 